\title{
Lessons of the month: Co-infection with SARS-CoV-2 and influenza $B$ virus in a patient with community-acquired pneumonia
}

\author{
Authors: Abigail Coutinho, ${ }^{A}$ Afrah Riaz, ${ }^{B}$ Annabel Makan, ${ }^{C}$ Emma Crawford, ${ }^{C}$ Devapriya Dev, ${ }^{C}$ Koottalai Srinivasan, ${ }^{C}$ \\ Nawaid Ahmad ${ }^{C}$ and Harmesh Moudgil ${ }^{C}$
}

\begin{abstract}
Why we only infrequently detect or report two or more respiratory viruses co-infecting an adult host is poorly understood. We report a rare case where influenza $B$ and SARS-CoV-2 caused viral pneumonia in a 74-year-old man diagnosed during the UK winter epidemic/pandemic for these organisms and discuss concepts of co-infection.
\end{abstract}

KEYWORDS: COVID-19, SARS-CoV-2, co-infection, influenza B, viral co-infection

DOI: $10.7861 /$ clinmed.2020-0598

\section{Case presentation}

A 74-year-old man, an ex-smoker (40 pack-years) with a background of chronic obstructive pulmonary disease (COPD), hypertension and type 2 diabetes, presented to his general practitioner with a 4 -day history of a non-productive cough, low-grade fever, myalgia and malaise. He was prescribed oral amoxicillin and prednisolone but, 2 days later, was admitted via the emergency department with new shortness of breath but no other evolving symptoms. His medications were metformin, losartan, and a Trelegy Ellipta (fluticasone furoate, umeclidinium and vilanterol) inhaler. He lived with his daughter who was a nurse but well and denied contact with confirmed COVID-19 patients and neither reported recent travel abroad. He had not been vaccinated against the winter flu.

Temperature at admission was $37^{\circ} \mathrm{C}$, blood pressure was $163 / 81$ $\mathrm{mmHg}$ with tachycardia at 110 beats per minute and tachypnoea at 35 breaths per minute; arterial blood gases confirmed hypoxic respiratory failure with peripheral oxygen saturations $86 \%$ on room air improving to $94 \%$ on supplemental nasal oxygen at $2 \mathrm{~L} / \mathrm{min}$. Blood tests revealed a mildly elevated C-reactive protein (CRP) at $54 \mathrm{mg} / \mathrm{L}(0-5)$, aminotransferase at $80 \mathrm{U} / \mathrm{L}(0-45)$, serum alkaline phosphatase at $147 \mathrm{U} / \mathrm{L}(40-120)$ and gamma glutamyl

Authors: ${ }^{\text {A }}$ core medical trainee, Princess Royal Hospital, Telford, UK; ${ }^{\text {B }}$ foundation year-1 doctor, Princess Royal Hospital, Telford, UK;

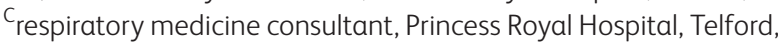
UK transferase at $375 \mathrm{U} / \mathrm{L}(0-75)$ but lactate dehydrogenase had not been measured. Total white cell count was normal but with mild lymphopenia at $0.9 \times 10^{9} / \mathrm{L}\left(1-4 \times 10^{9} / \mathrm{L}\right)$. Chest auscultation revealed global bilateral expiratory wheeze with no added crackles and chest X-ray (CXR) on admission did not show any focal changes. Differentials included infective exacerbation of COPD, influenza with the UK winter season, and COVID-19 with the evolving pandemic. The patient was treated with nebulised bronchodilators, intravenous hydrocortisone and oral doxycycline but there was continued decline with oxygen requirements increased to $4 \mathrm{~L} / \mathrm{min}$ at 8 hours and then to $6 \mathrm{~L} / \mathrm{min}, 24$ hours later. Blood tests showed a further small rise in CRP to $72 \mathrm{mg} / \mathrm{L}$ and worsening lymphopenia to $0.5 \times 10^{9} / \mathrm{L}$. Polymerase chain reaction (PCR) assay detected influenza $B$ and oseltamivir (antiviral therapy) was started.

The oxygen demand increased to $15 \mathrm{~L} / \mathrm{min}$ and continuous positive airway pressure (CPAP) was added. A repeated CXR (Fig 1) newly showed nodularity and ground-glass opacifications bilaterally throughout the lower zones, leading to a diagnosis

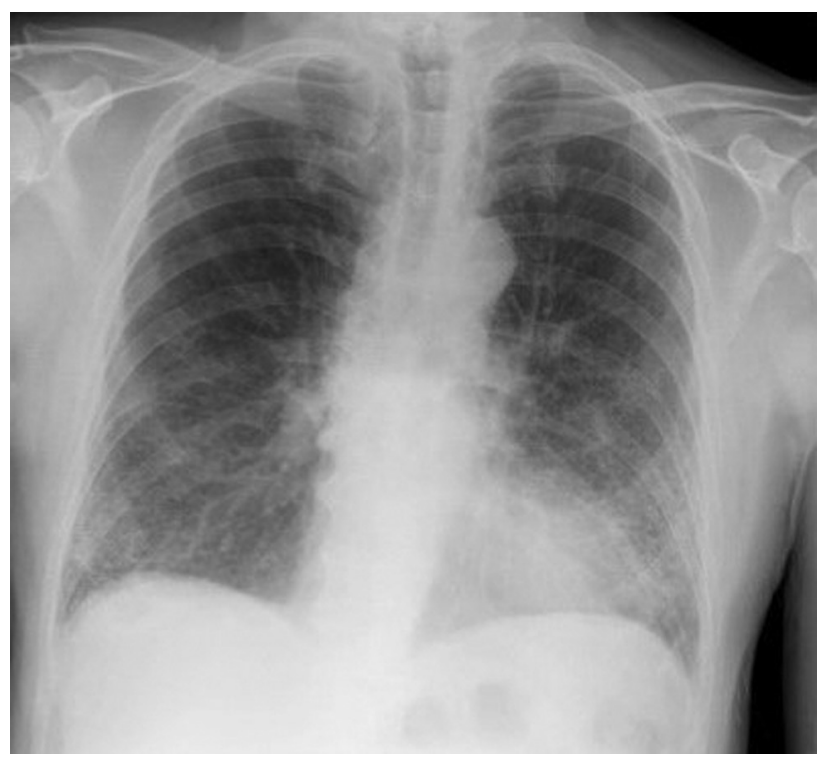

Fig 1. Chest $\mathrm{X}$-ray showing nodularity and ground-glass opacifications bilaterally throughout the lower zones. 
of a community acquired pneumonia (CAP). Antibiotics were empirically changed to azithromycin. Three days later, the reverse transcriptase PCR assay for SARS-CoV-2 from a nasopharyngeal swab specimen taken at admission tested positive. The patient tolerated CPAP well and over the next 6 days was successfully weaned off all respiratory support. No bacterial infections were detected.

\section{Discussion}

'Co-infection' in virology describes simultaneous infection of a cell or organism by separate viruses; infection with both influenza A and the coronavirus SARS-CoV-2 has recently been described. ${ }^{1}$ Our case is potentially unique in the literature, detecting the less common influenza B and SARS-CoV-2; illness in either infection starts with non-specific upper respiratory tract symptoms making them more difficult to distinguish clinically and similarly from some other seasonal viral infections including influenza A, parainfluenza and respiratory syncytial virus (RSV). Although a disabling illness in patients more severely affected with COVID-19, infections with either pathogen may progress onto an adult respiratory distress syndrome which, with influenza viruses, is usually when complicated by secondary bacterial pneumonias; Streptococcus pneumonae, Haemophilus pneumonae and Staphylococcus aureus are most commonly implicated.

Most cases of CAP are caused by a single pathogen but increasingly concurrent infections with both bacterial and viral pathogens are being studied; publications in adults suggest $13.6 \%$ diagnosed with CAP and $21.9 \%$ with severe disease have a mixed infection associated with higher morbidity and mortality rates. ${ }^{2}$ Mechanisms include a disruption of the epithelial barrier, upregulation of cell surface binding sites for bacteria, or dysfunction of the immune system. ${ }^{3,4}$ The severity of illness depends on the strain of virus and serotype/strain of bacteria, the lag between viral infection and bacterial exposure, and density of bacterial colonisation. To date, however, with the exception to bacterial mixed infection with influenza, the role of antiviral agents in other mixed bacterial and viral infected patients with CAP is not established. . $^{2}$

Research sequencing genetic material from multiple strains of rhinovirus has suggested co-infection within different strains is common and provides viruses the opportunity to mutate further; the same is also true for ribonucleic acid (RNA) viruses, such as influenza, where high nucleotide substitution and poor proofreading leads to the accumulation of errors in newly synthesized RNA strands. ${ }^{4}$ Although residual viral fragments from infections over the preceding 5-6 weeks may affect detection rates, research in children has reported viral co-infection in $61.81 \%$ of patients with positive samples; findings showed two viruses in $36.36 \%$, three in $16.10 \%$ and four or more in $9.35 \% .{ }^{6}$ Common infections include rhinovirus, adenovirus, coronaviruses and RSV.

There are no underlying clinical differences between patients with respiratory infections caused by a single agent and those caused by multiple viruses. The clinical course with more than one viral infection invading a host is less understood and inconsistent.
With respiratory viruses, although the illness generally lasts longer, the severity is more dictated by the individual's immune cytokine and chemokine response. The immune response to the first virus modifies disease severity of a subsequent acquired virus through viral interference mediated via interferons (IFNs) where one virus competitively suppresses replication of other viruses; conversely there may be an increase in viral replication, or even no effect, where viruses coexist (accommodation). ${ }^{7}$ With non-IFN-mediated or intrinsic interference, there is competition between viruses for metabolites, replication sites and other host factors. ${ }^{7}$ Translated into clinical outcomes, compared with infection with multiple viral infections, illnesses with a single virus tend to have an increased risk of oxygen requirement, increased number of admissions and duration of stay in hospital or to intensive care. ${ }^{8}$

\section{Conclusion}

This case is potentially unique in the literature and reports findings in a patient diagnosed to have co-infection with influenza $B$ and the SARS-CoV-2. Host co-infection with two or more respiratory viruses or strains occurs more frequently than usually reported but the subsequent clinical course does not necessarily intensify and this may, in part, be because of a muted host immune response where there is co-infection.

\section{References}

1 Wu X, Cai Y, Huang X, Yu X et al. Co-infection with SARS-Cov-2 and influenza $A$ virus in patient with pneumonia, China. Emerg Infect Dis 2020;26:1324-6.

2 Lim Y, Kweon O, Kim H, Kim T, Lee M. Impact of bacterial and viral coinfection in community-acquired pneumonia in adult. Diagn Microbiol Infect Dis 2019;94:50-4.

3 Bosch A, Biesbroek G, Trzcinski K, Sanders EA, Bogaer D. Viral and bacterial interactions in the upper respiratory tract. PLoS Pathog 2013:9;e1003057

4 Morris D, Cleary D, Clarke S. Secondary bacterial infections associated with influenza pandemics. Front Microbiol 2017;8:1041.

5 Chertow D, Memoli M. Bacterial coinfection in influenza: a grand rounds review. JAMA 2013:309;275-82.

6 Martínez-Roig A, Salvadó M, Caballero-Rabasco M. Viral coinfection in childhood respiratory tract infections. Arch Bronconeumol 2015; 51:5-9.

7 Kumar N, Sharma S, Barua S, Tripathi BN, Rouse BT. Virological and immunological outcomes of coinfections. Clin Microbiol Rev 2018;31:e00111-17

8 Martin E, Kuypers J, Wald A, Englund JA. Multiple versus single virus respiratory infections: viral load and clinical disease severity in hospitalized children. Influenza Other Respir Viruses 2012;6:71-7.

Address for correspondence: Dr Abigail Coutinho, The Princess Royal Hospital, Apley Castle, Apley, Telford TF1 6TF, UK. Email: abigail.coutinho3@nhs.net 